\title{
Comparative study of limited-range wavelength conversion policies for asynchronous optical packet switching
}

\author{
Kaan Dogan \\ Department of Electrical and Electronics Engineering, Bilkent University, \\ Ankara, Turkey \\ Yavuz Gunalay \\ Faculty of Business Administration, Bilkent University, \\ Ankara, Turkey \\ Nail Akar \\ Department of Electrical and Electronics Engineering, Bilkent University, \\ Ankara, Turkey \\ akar@ee.bilkent.edu.tr
}

Received October 3, 2006; revised November 16, 2006;

accepted November 22, 2006; published January 19, 2007 (Doc. ID 75693)

\begin{abstract}
We study an asynchronous optical packet (OP)-switching node equipped with a number of limited range (LR) wavelength converters shared per output link. We study both circular and noncircular LR-wavelength-conversion schemes. A wavelength conversion policy governs the selection of the outgoing wavelength for an OP if the incoming wavelength is in use. Through simulations, we show that the so-called far-conversion policy for which the OP is switched onto the farthest available wavelength in the tuning range, outperforms the other policies we studied. We point out the clustering effect in the use of wavelengths to explain this phenomenon. We also provide an approximate analytical method to find the packet-blocking probability in circular-type LR-wavelengthconversion systems. Based on the simulation results, the approximate method appears to lead to a lower bound for blocking probabilities for all the conversion policies we study. (C) 2007 Optical Society of America

OCIS codes: $060.4250,000.5490$
\end{abstract}

\section{Introduction}

Optical data transmission over fiber lines with the wavelength division multiplexing (WDM) technology helps network operators to cope with rapidly increasing traffic demands. WDM allows multiplexing of several wavelength channels on a single fiber, and WDM systems with 32 or 64 channels, each operating at $10 \mathrm{Gbit} / \mathrm{s}$ for a total capacity of 320 or $640 \mathrm{Gbit} / \mathrm{s}$ over a single fiber, are common today. Two packet-based optical-switching paradigms have been proposed to make more efficient use of bandwidth (as opposed to circuit-based networks): optical packet switching (OPS) [1,2] and optical burst switching (OBS) [3,4]. OPS requires line-rate header parsing and is viewed as a longer-term solution due to the current technological limitations in packet header processing [2]. OBS, on the other hand, eliminates the need for header parsing by segregating the control and data planes. In OBS, the reservation request for a burst is signaled out of band by the use of a burst control packet, which is processed all in the electronic domain. However in OBS, the payload is transported as a burst end-to-end all in the optical domain. The scope of this study encompasses both OPS and OBS since the data planes of these two technologies are very similar, but in this paper, for the sake of convenience, we will use the common term "(optical) packet" and "(optical) packet switching" to refer to a packet and/or burst and the data planes of OPS and/or OBS, respectively.

In synchronous (i.e., time-slotted) OPS networks, packet lengths are fixed, and packets are assumed to arrive at slot boundaries. Such models are relatively difficult 
to implement due to the need for costly synchronization equipment. In asynchronous (i.e., unslotted) networks, optical packet (OP) lengths are variable and packet arrivals need not be aligned. Therefore, there is no need for costly synchronization equipment. Moreover, asynchronous packet switching (PS) is a natural fit for supporting asynchronous information processing networks with variable-sized data packets. Therefore, we focus on asynchronous OPS in this study.

One of the major issues in OPS networks is contention, which arises as a result of two or more incoming packets contending for the same output wavelength. Contention is resolved either in wavelength domain by wavelength converters, in time domain by fiber delay lines (FDLs), or in space domain by deflection routing [3]. If contention cannot be resolved by any one of the proposed techniques, then a number of the contending packets would be blocked. In this paper, we focus on contention resolution using only tunable wavelength converters (TWCs). In full wavelength conversion (FWC), we have a converter dedicated to each wavelength channel. In partial wavelength conversion (PWC), we have converter sharing among a number of wavelength channels. In this case, OPs may not be switched toward their destination and therefore are blocked when all converters are occupied despite the availability of free wavelength channels on the output link. In PWC, TWCs may be collected as a single converter pool for converter sharing across all fiber lines, which is referred to as the share-per-node (SPN) architecture [5]. On the other hand, the share-per-link (SPL) architecture allows dedicated TWC banks for each output fiber link [5]; see Fig. 1 for a descriptive picture of the SPL architecture. The cost-effective SPL solution has its advantages of low-complexity switching matrix, significant performance, and TWC saving gain compared with other alternatives [5], in addition to its amenability to exact stochastic analysis [6]. Another crucial issue in wavelength conversion is whether there is a specified range of wavelengths that a given wavelength can be converted to. Full-range tunable wavelength converters (FR-TWCs) do not have any tuning range limit, and they can convert an incoming wavelength to any other wavelength. In limited-range (LR) wavelength conversion, a packet arriving on a wavelength can be converted to a fixed set of wavelengths above and below the original wavelength, and such TWCs are called limited-range tunable wavelength converters (LR-TWCs) [7]. For LR-TWCs, the conversion degree $d$ is defined as the total number of wavelengths available on both sides of the original wavelength, and therefore, an incoming OP can either be switched to the destination fiber on the incoming wavelength if available or be converted to one of the $d$ wavelengths in its physical neighborhood if one of those is idle. Two different conversion schemes have been introduced on the basis of the neighboring relationship for the wavelengths at the boundaries, namely, circular- and noncircular-conversion schemes [8].

The focus of the current paper is the performance of an asynchronous OPS employing PWC on an SPL basis with the shared TWCs being of LR type; see Fig. 1. Due to the way converters are shared, each outgoing link can be studied independently. We

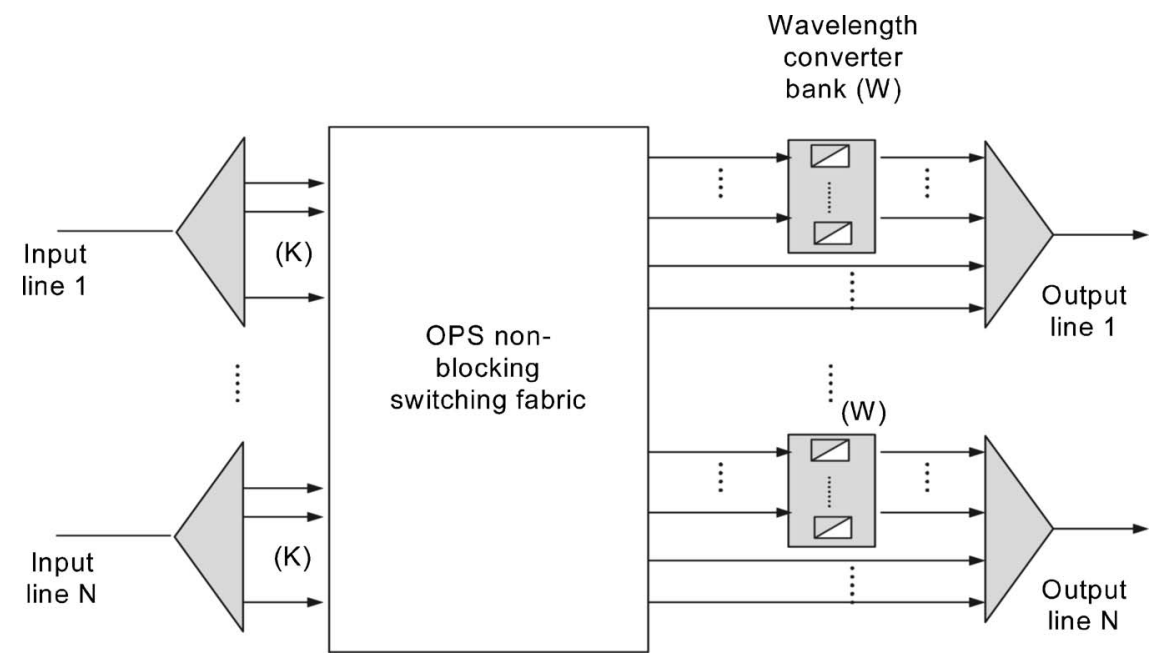

Fig. 1. General architecture of an OPS node with $N$ fiber I/O lines, $K$ wavelength channels on each fiber line, and a bank of wavelength converters of size $W$ shared per output link. 
assume that $N$ is large enough so that the packet traffic destined to a specified outgoing link can be modeled with the Poisson process with rate $\lambda$. The number of channels on the given link is denoted by $K$, and a converter bank of size $0<W \leq K$ is assumed to be available for that link. As a usual assumption, the wavelength distribution of the incoming request is assumed to be uniform among all the wavelengths. We also adhere to the exponential packet length distribution assumption, which is common in the literature where the mean packet length is denoted by $1 / \mu$. The system load is denoted by $\rho=\lambda / \mu K$. An exact and efficient analytical procedure is proposed for the same problem for the case of FR-TWCs in [6,9]. However, a similar exact analysis does not seem to be plausible for the case of LR-TWCs due to the way conversion ranges overlap. Moreover, random conversion to any of the available wavelengths in the case of FR-TWCs provides the best results because of the uniform distribution of the incoming wavelengths. However, conversion policies substantially impact the performance for the case of LR-TWCs. The goal of this paper is to study different simple-toimplement wavelength conversion policies for asynchronous PS with circular LR-TWCs. On the other hand, we give an analytical method based on [6] to give an approximation for the packet-blocking probability. The approximate method involves the exact analysis of an auxiliary model that captures part of the complex system dynamics. Finally, we compare the results of the circular-conversion case with those of the noncircular-conversion case using simulations.

Existing LR-wavelength-conversion studies focus on either circuit-switched or packet and/or burst-switched networks. A circuit-switched WDM-based optical network uses a two-way reservation scheme where a source node sends out a control packet to make a reservation and then waits for an acknowledgment from the destination before data transmission. Given an optical network with LR-TWCs in place and upon a lightpath establishment request from a source to a destination the problem reduces to finding a free wavelength on each link on the path to the destination with the succesive wavelengths satisfying LR-wavelength-conversion constraints at each hop. The analysis of LR-wavelength conversion is first addressed in [10], which is limited to some specific network topologies. An approximate stochastic analysis for circuit-switched optical networks with arbitrary topology and fixed routing using circular LR-TWCs and for Poisson arrivals is given in [11]. On the other hand, OPS, and/or OBS architectures rely on the one-way reservation scheme. In the one-way reservation model, outgoing wavelength assignments for OPs need to be made locally by a switching node without a priori information on the free wavelengths available at the downstream links. Therefore, LR-wavelength-conversion problems are different for the circuit- and packet-based switching paradigms. Most of the literature on LR-wavelength conversion in OPS assumes synchronous networks. In this case, all the OPs aligned at slot boundaries are treated together, and an optimum scheduling is sought to minimize the packet loss probability. The scheduling algorithm provides a policy to decide on which packets to transmit, which packets to be wavelength converted, and which output wavelength channels to assign to incoming packets. References $[8,12]$ study optimum scheduling algorithms to maximize the throughput of the switch, and they provide analytical models to evaluate the performance of the switch. In the case of asynchronous PS, the scheduling problem disappears since the switch will handle OPs one at a time. In [13], a product form solution is given for the special cases of $d=2$ and $d=4$ for on-off traffic, whereas an approximation technique is presented for more general $d$. However, to the best of our knowledge, the impact of wavelength-conversion policies on packet-blocking performance in asynchronous PS systems with the well-known Poisson arrival model has not been studied before.

The outline of the paper is as follows. We introduce a number of simple-toimplement wavelength-conversion policies in Section 2. We provide an approximate analysis method to find the blocking probabilities for the SPL LR-TWC architecture in Section 3 for the circular-conversion case. Numerical results are presented in Section 4 for both circular and noncircular cases. We conclude in Section 5.

\section{Conversion Policies for Limited-Range Tunable Wavelength Converters}

In this paper, we study two LR-wavelength-conversion schemes, namely, circular and noncircular schemes, which will be described next. Let us assume $K$ wavelengths, 
indexed as $i=0,1, \ldots, K-1$. In circular conversion, we assume the wavelengths are wrapped around to form a circle so that the wavelengths at the boundaries, i.e., 0 and $K-1$, become neighbors. The circular-type LR-wavelength-conversion scheme is depicted in Fig. 2 for which the conversion range of an incoming wavelength $i, 0 \leq i$ $\leq K-1$, denoted by $R_{i}$, is given by the following wavelength set:

$$
R_{i}=\left\{\bmod \left(i-\frac{d}{2}, K\right), \ldots, \bmod (i-1, K), \bmod (i+1, K), \ldots, \bmod \left(i+\frac{d}{2}, K\right)\right\} .
$$

In the above definition, $\bmod (a, b)$ is the remainder on division of $a$ by $b$ for given integers $a$ and $b$. Revisiting Fig. 2(a) for the case of $d=4$, the incoming boundary wavelength 0 can be converted to the set $\{K-2, K-1,1,2\}$, and the incoming wavelength $i$ can be converted to the set $\{i-2, i-1, i+1, i+2\}$ assuming $2 \leq i \leq K-3$. Circular-type LR conversion preserves the symmetry among wavelengths and is therefore the preferred conversion scheme when analysis is sought. On the other hand, in noncirculartype LR conversion, we do not allow wrap-around and the conversion ranges for wavelengths close to the boundaries are reduced in size. The conversion range definition for noncircular-type conversion for wavelength $i, 0<i<K-1$, is written as

$$
R_{i}=\left\{\max \left(i-\frac{d}{2}, 0\right), \ldots, \max (i-1,0), \min (i+1, K-1), \ldots, \min \left(i+\frac{d}{2}, K-1\right)\right\} .
$$

On the other hand, it is clear that $R_{K-1}=\{K-1-d / 2, K-d / 2, \ldots, K-2\}$, and $R_{0}$ $=\{1,2, \ldots, d / 2\}$. In Fig. 2(b), we provide the conversion ranges for the boundary wavelengths as well as a wavelength $i$ far from the boundaries when the conversion degree $d=4$. Although this conversion scheme might be more realistic, it seems very difficult to analytically address the asymmetry among the wavelengths. In this paper, we study both circular- and noncircular-conversion schemes using simulations but provide an approximate analysis method only for the circular-conversion case.

The stochastic analysis carried out in [6] involves FR-TWCs in which a wavelength is selected randomly out of the set of idle wavelengths in case the incoming wave-

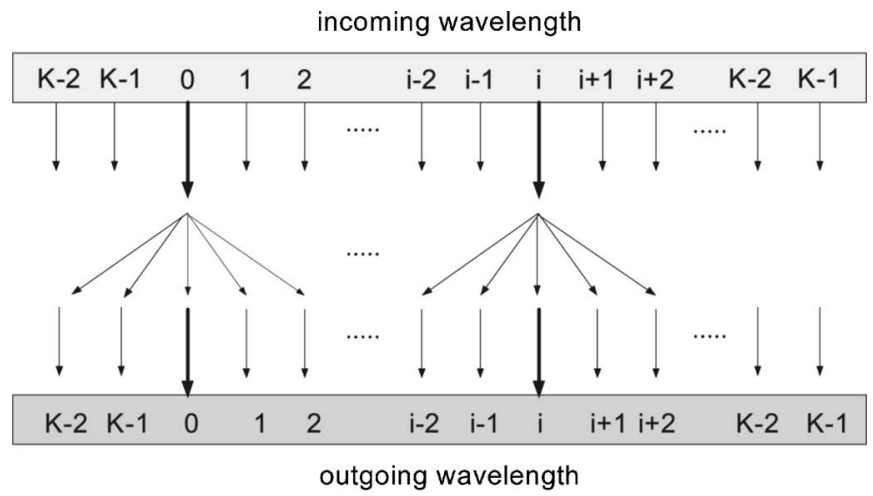

(a) circular scheme

incoming wavelength

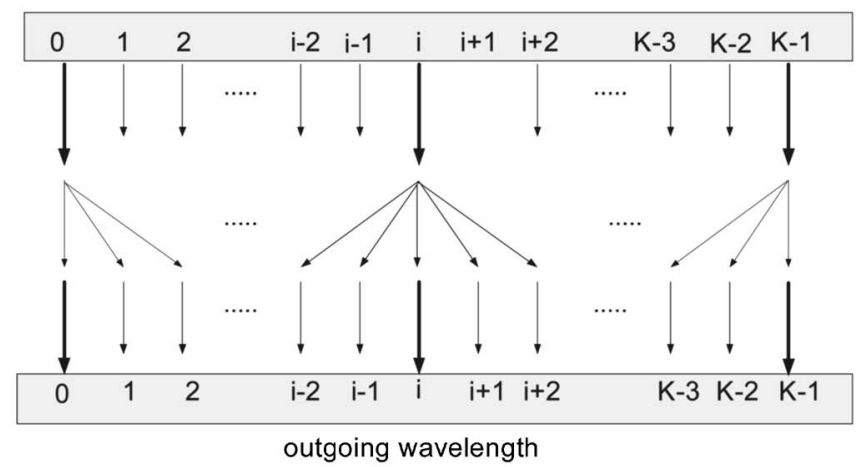

(b) non-circular scheme

Fig. 2. (a) Circular scheme, (b) noncircular conversion scheme depicted for $d=4$ for the boundary incoming wavelength 0 and a wavelength $i$ far from the boundaries. 
length is occupied. This randomized policy is best for FR-TWCs when the wavelength of the incoming packets is uniformly distributed. However, such a randomized policy has drawbacks in the case of LR-TWCs even for the case of uniformly distributed incoming wavelengths. We explain this phenomenon by the following scenario. Consider the arrival instance of an OP $x$ whose incoming wavelength $i$ is occupied in the outgoing link and therefore the packet requires wavelength conversion. This shows that either a packet had arrived on the same wavelength $i$ and it is still being served, or a packet had arrived on a different wavelength $j$ but converted to $i$ since $j$ was occupied. Since conversions take place within a range of the incoming wavelength, the probability of packet $x$ to find its conversion range fully occupied is larger than the full occupancy probability of an arbitrarily selected set of $d$ wavelengths other than the wavelength $i$. Equivalently, there is a positive spatial correlation between the status of two neighboring wavelengths, and consequently occupied wavelengths tend to appear clustered in time at the embedded epochs of a packet finding its own wavelength occupied. This phenomenon does not occur for the case of FR-TWCs. The so-called clustering phenomenon obviously has a detrimental impact on blocking performance. On the other hand, the clustering effect can be mitigated by appropriate wavelength conversion policies. We study the following three simple-to-implement wavelength-conversion policies at the instance of a packet arrival whose incoming wavelength is occupied.

- Random Conversion: The outgoing wavelength is selected randomly from the set of idle wavelengths in the range.

- Near Conversion: We choose the nearest available wavelength from the set of idle wavelengths in the conversion range, and if there exist two such wavelengths, one of them will be selected in random. However, such a policy works in favor of the clustering effect relative to the random-conversion policy.

- Far Conversion: In this policy, the farthest available wavelength is selected from the set of idle wavelengths in the conversion range. If there exist two such wavelengths, one of them will be selected in random. Obviously, this policy counteracts the clustering effect.

We explain the clustering effect through a simulation study for which both circularand noncircular-wavelength-conversion schemes are to be used for a scenario corresponding to $K=33, W=16, d=8$, and a system load of $\rho=0.3$. We assume that the wavelengths are indexed as $0, \ldots, 32$. We concentrate our attention on the middle wavelength numbered 16 and mark the instances for which packets arriving on wavelength 16 find that wavelength in use. We note the occupancy probability of the other wavelengths at these embedded epochs. We first provide such conditional occupancy probabilities in Fig. 3(a) for the circular scheme. In this case, all conversion policies tend to produce a wavelength occupancy probability histogram clustered within the conversion range of the incoming wavelength. However, such clustering is most dominant in the near-conversion policy.

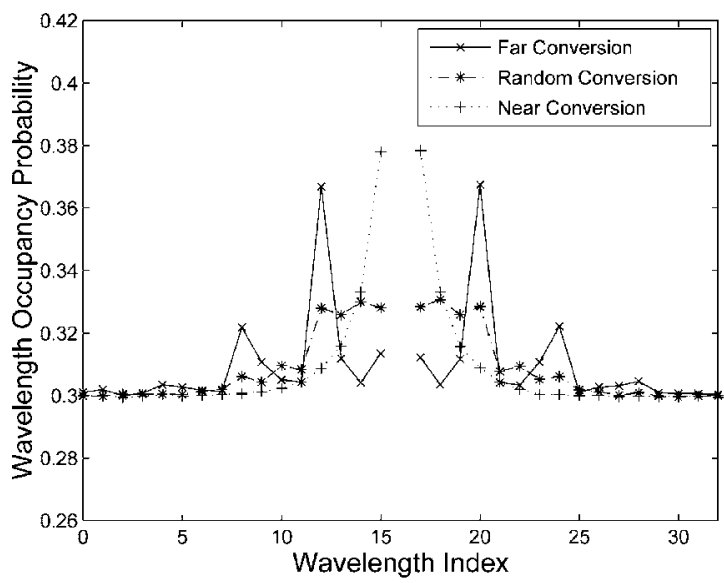

(a) circular case

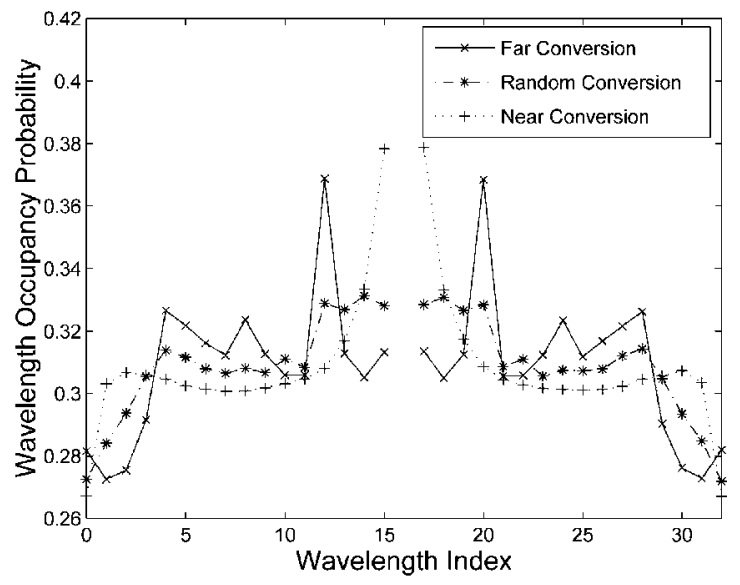

(b) non-circular case

Fig. 3. Occupancy probabilities of wavelengths conditioned upon an arriving packet on the midwavelength, i.e., wavelength 16 , finding its own wavelength occupied in (a) circular case, (b) noncircular-case LR-wavelength conversion. 
We also note that the far-conversion policy produces occupancy probability peaks at wavelengths 12 and 20 (i.e., $d / 2$ units away from the incoming wavelength), which are still within the conversion range of wavelength 16 . However, the second- and thirdorder harmonics at wavelengths 8 and 24, and 4 and 28, respectively, generated by the far-conversion policy, appear to increase the probability of an arriving packet on wavelength 16 to find at least one idle wavelength in its conversion range.

We repeat the same experiment for the noncircular scheme for which the results are depicted in Fig. 3(b). The conditional wavelength occupancy probability histogram appears to be similar to that of the circular scheme with an exception at the thirdorder harmonics at approximately wavelengths 4 and 28. At these wavelengths, a sharp increase in the occupancy probabilities is followed by sharp decreases. This can be explained by the reduced tuning ranges near the boundary wavelengths.

All the three LR-wavelength-conversion policies, independent of the conversion scheme (i.e., for both circular and noncircular cases), obviously result in identical performance for the particular cases $d=2$ and $d=K-1$. In the former case, there are only two adjacent candidate wavelengths for conversion, and all three policies collapse to a random-conversion policy. The case of $d=K-1$ reduces to full-range conversion for which we do not observe clustering. The goal of the current paper is to show if there is a notable difference among the three conversion policies for $2<d<K-1$, i.e., moderate degree of conversion.

\section{Analytical Model}

An exact stochastic analysis for LR-wavelength conversion under the three proposed policies does not appear to be plausible even for the circular-conversion scheme. However, as a reference, we propose the following simple auxiliary model that captures part of the system dynamics. In this auxiliary model, the conversion range is not the actual $d / 2$ neighborhood of the incoming wavelength but instead a set of arbitrarily selected $d$ wavelengths at each time conversion is to take place. Although this system is very different than the original circular-type LR-wavelength-conversion system, we use this model for its amenability to exact stoshastic analysis. Moreover, this auxiliary model captures the impact of the degree of conversion. However, the model falls short of describing the clustering effect, which was shown in the previous section to work against packet success. Therefore, we expect to see reduced packet-blocking probabilities as a result of the auxiliary model when compared with the original system. One of the goals of this paper is to show the level of effectiveness of this auxiliary model in approximating the behavior of circular-type LR-wavelength-conversion systems.

We present below the exact stochastic analysis method for the auxiliary model based on the work [6]. For this purpose, we first study the auxiliary model for the case of PWC, i.e., $W<K$, which will be followed by the study of the simpler FWC case, i.e., $W=K$.

\section{A. Partial Wavelength Conversion}

In this case, an incoming $\mathrm{OP}$

- is forwarded without conversion if its incoming wavelength is idle on the outgoing link;

- is directed to the outgoing link after wavelength conversion if there exist both an available TWC and an idle wavelength in the conversion range, which, in this auxiliary model, is a randomly selected set of $d$ wavelengths other than the incoming wavelength;

- is blocked otherwise.

Under these assumptions, let $i(t)$ and $j(t)$ denote the number of wavelength channels and the number of TWCs that are in use at time $t$, respectively. The process $X(t)=\{(i(t), j(t)): t \geq 0\}$ is a continuous time Markov process on the state space $S$ $=\{(i, j): 0 \leq i \leq K, 0 \leq j \leq \min (i, W)\}$. To show this, let us assume that the process is in some state $(i, j), 0 \leq i<K, 0 \leq j \leq \min (i, W)$ at time $t$. If a new packet arrives in the interval $(t, t+\delta)$, which occurs with probability $\lambda \delta+o(\delta)$ [i.e., $\lim _{\delta \rightarrow 0} o(\delta) / \delta=0$ ] ([14], p. $48)$, there are four possibilities: 
- the incoming wavelength of the packet is not currently used on the link, which occurs with probability $(K-i) / K$, and the packet will be accepted, and the process will jump to $(i+1, j)$ at time $t+\delta$,

- that wavelength is already used, which occurs with probability $i / K$

- then if $j=W$, then the packet will be blocked because the converter pool is busy, leading to no state change,

- else if $j<W$ and all of the wavelength channels that are in the conversion range of the incoming wavelength are occupied, then the packet will be blocked. The probability of the occupancy of all the $(i-1)$ wavelengths in the tuning range in this case is expressed as

$$
p_{i}= \begin{cases}\frac{\left(\begin{array}{c}
K-d-1 \\
i-1-d
\end{array}\right)}{\left(\begin{array}{c}
K-1 \\
i-1
\end{array}\right)}=\frac{i-1}{K-1} \frac{i-2}{K-2} \cdots \frac{i-d}{K-d}, & \text { if } i \geq d+1 \\
0, & \text { if } i \leq d\end{cases}
$$

- else the conversion range has at least one free wavelength available, then the packet will be admitted onto one of the available wavelengths randomly using one of the free converters, and the process will make a transition to state $(i+1, j+1)$ at time $t+\delta$.

Assume now that the process $X(t)$ is currently in some state $(i, j), 0<i \leq K, 0 \leq j$ $\leq \min (i, W)$ at time $t$. If a packet departs in the interval $(t, t+\delta)$, which occurs with probability $i \mu \delta+o(\delta)$, then there are two possibilities:

- a TWC was used for this packet, which occurs with probability $j / i$, and the process $X(t)$ will jump to state $(i-1, j-1)$ at time $t+\delta$;

- a TWC was not used at all for this departing packet, which occurs with probability $(i-j) / i$, and the process $X(t)$ will make a transition to state $(i-1, j)$ at time $t+\delta$.

When the process $X(t)$ is in state $(0,0)$, then there cannot be any departures. It is thus clear that the process $X(t)$ is a continuous time Markov chain (CTMC), and the infinitesimal generator of the CTMC possesses a block-tridiagonal form if the states are properly enumerated as in [6]. A numerically stable and efficient solution procedure, the so-called block-tridiagonal lower triangular-upper triangular factorization algorithm can then be used to find the stationary solution of the underlying CTMC while taking advantage of the block-tridiagonal structure of the generator ([15], pp. 174-175).

For obtaining the packet-blocking probabilities, we observe that a new packet arrival is blocked if

- the Markov chain resides in $(K, j), 0 \leq j \leq W$ (i.e. all wavelength channels are in use),

- the Markov chain resides in state $(i, W), W \leq i<K$ (i.e. all converters are in use), and the incoming wavelength is occupied (this occurs with probability $i / K$ ),

- the Markov chain resides in states $(i, j), d<i<K, 0 \leq j<W$, the incoming wavelength is occupied, and the conversion range is fully occupied, which occurs with probability:

$$
\frac{i\left(\begin{array}{c}
K-d-1 \\
i-1-d
\end{array}\right)}{K}
$$

By taking advantage of the Poisson arrivals see time averages property ([16], pp. 221-222), the packet-blocking probability $P_{b}$ of the auxiliary model can be written as

$$
P_{b}=\underbrace{\sum_{j=0}^{W} x_{K, j}}_{\text {I }}+\underbrace{\sum_{i=W}^{K-1} \frac{i}{K} x_{i}, w}_{\text {II }}+\underbrace{\sum_{i=d+1}^{K-1} \sum_{j=0}^{W-1} x_{i, j} \frac{i}{K} \frac{\left(\begin{array}{c}
K-d-1 \\
i-1-d
\end{array}\right)}{\left(\begin{array}{c}
K-1 \\
i-1
\end{array}\right)}}_{\text {III }},
$$


where $x_{i}=\left(x_{i, 0}, x_{i, 1}, \ldots, x_{i, W}\right)$ for $i \geq W, x_{i, j}$ is the steady-state probability of having $i$ wavelengths and $j$ TWCs occupied. The three terms I, II, and III in Eq. (5) correspond to the probability of loss stemming from lack of capacity, lack of converters, and the fullness of the tuning range. The term $\mathrm{I}$ is dominant when the system load is high. In lightly loaded systems with few shared converters, it is term II that dominates the overall performance. In these two cases, a certain LR-wavelength-conversion scheme will not have much of an impact on overall performance. However, for low-to-moderate loads and with FWC-like converter sharing, i.e., $W$ is close to $K$, term III dominates the other two, and which conversion policy to use from an overall performance standpoint matters.

\section{B. Full Wavelength Conversion}

In the auxiliary model of the previous subsection, $W<K$ is assumed. In the FWC case, the number of converters equals the number of channels, and there is no chance of packet blocking due to unavailability of converters, and the model further simplifies to an $\mathrm{M} / \mathrm{M} / \mathrm{K} / \mathrm{K}$ loss system with probabilistic server assignment. In this system, if a packet finds the corresponding channel busy, it can be converted to a neighboring channel (within the given range $d$ ) unless the range is full. We again introduce the auxiliary model here, and we assume that a packet can be converted to $d$ other randomly selected wavelengths as opposed to one in the actual tuning range. In this case, the auxiliary system behaves as a finite-state birth and death process ([14], pp. 105106) where $\lambda_{j}$ and $\mu_{j}$ are the birth and death rates at state $j$, respectively, and

$$
\begin{gathered}
\lambda_{j}=\left\{\begin{array}{ll}
\lambda, & j=0, \ldots, d \\
\left(1-q_{j}\right) \lambda, & j=d+1, \ldots, K-1
\end{array},\right. \\
\mu_{j}=j \mu, \quad j=1, \ldots, K,
\end{gathered}
$$

where

$$
q_{j}=(j / K)\left(\begin{array}{c}
K-d-1 \\
j-1-d
\end{array}\right) /\left(\begin{array}{c}
K-1 \\
j-1
\end{array}\right), d+1 \leq j \leq K,
$$

is the joint probability of an arriving packet finding its incoming wavelength occupied and $d$ randomly selected wavelengths busy. We solve the equilibrium balance equations for this birth-death process, which yields the steady-state probabilities $\pi_{j}$ of finding $j$ channels busy:

$$
\pi_{j}=\left\{\begin{array}{ll}
\frac{\rho^{j}}{j^{!}} \pi_{0}, & j=1, \ldots, d+1 \\
\left(\begin{array}{ll}
j-1 \\
\left.\prod_{k=d+1}\left(1-q_{k}\right)\right)
\end{array}\right) \frac{\rho^{j}}{j !} \pi_{0}, & j=d+2, \ldots, K
\end{array},\right.
$$

where

$$
\pi_{0}=\left[1+\sum_{j=1}^{d+1} \frac{\rho^{j}}{j^{!}}+\sum_{j=d+2}^{K}\left(\underset{k=d+1}{j-1}\left(1-q_{k}\right)\right) \frac{\rho^{j}}{j^{!}}\right]^{-1} .
$$

Finally, the packet-blocking probability can simply be expressed as

$$
P_{b}=\sum_{k=d+1}^{K} q_{k} \pi_{k} \text {. }
$$

\section{Numerical Results}

In this study, we obtain the packet-blocking probabilities for the three wavelengthconversion policies by simulations. We also solve for the auxiliary model analytically using the technique described in the previous section for the circular scheme. The goal of this simulation example is to comparatively study the three policies and test the effectiveness of the auxiliary model. Without loss of generality, the mean packet length $1 / \mu$ is normalized to unity in all numerical examples presented below. Each simulation result is obtained by averaging ten independent runs. We do not present 


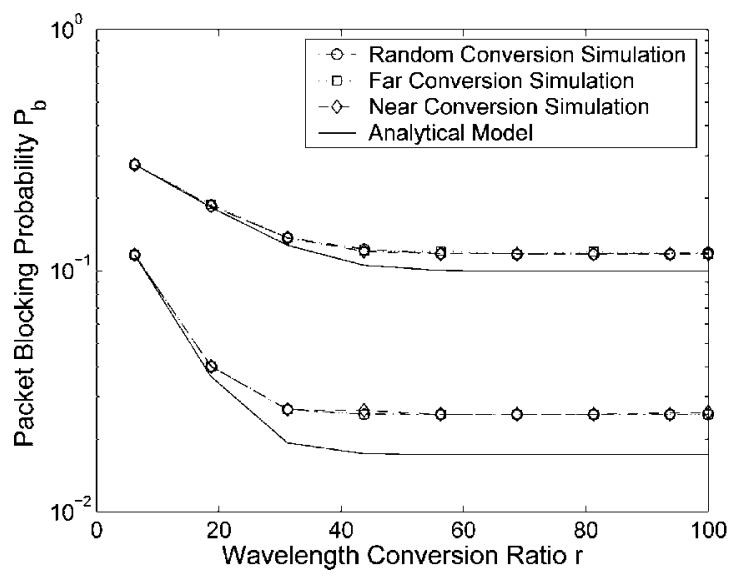

(a) $d=2$

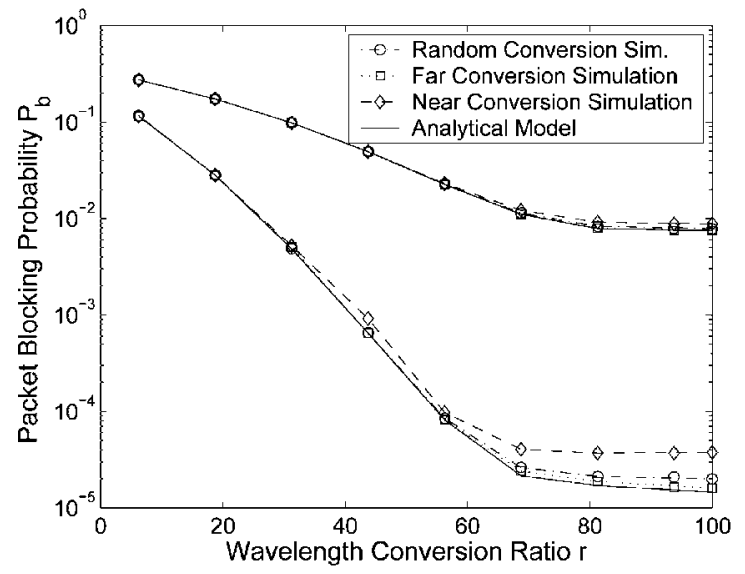

(c) $d=10$

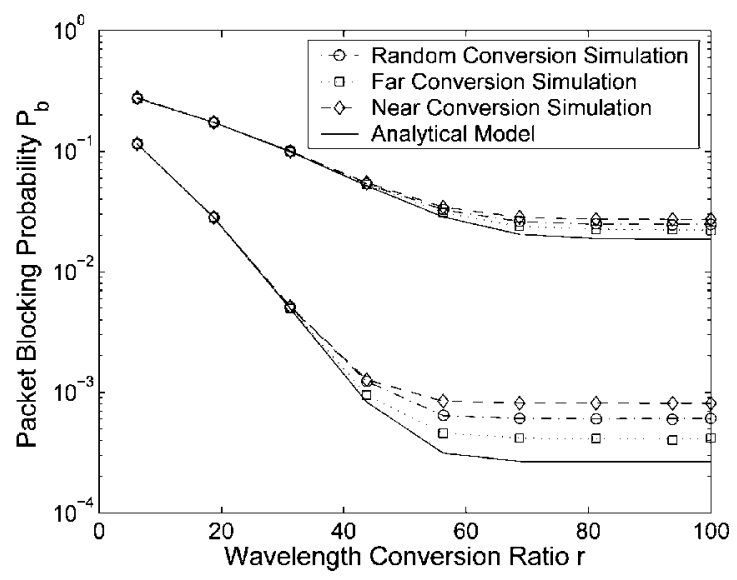

(b) $d=6$

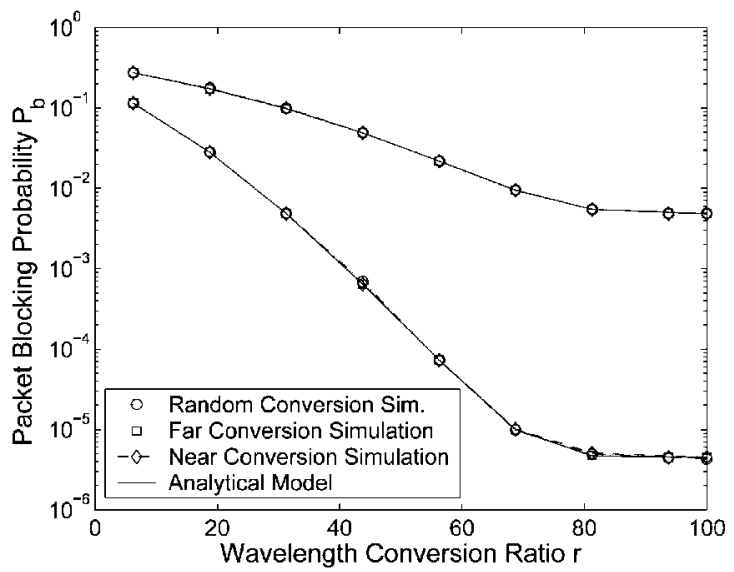

(d) $d=14$

Fig. 4. Blocking probabilities for $K=16$ with respect to $r$ for two different values of $\rho=0.25$ and $\rho$ $=0.5$ when (a) $d=2$, (b) $d=6$, (c) $d=10$, and (d) $d=14$.

blocking probabilities less than $10^{-9}$. We use percent ratios in this paper, therefore we define the wavelength-conversion ratio $r=100 \mathrm{~W} / \mathrm{K}$ and the tuning-range ratio $\gamma$ $=100 \mathrm{~d} / \mathrm{K}$.

In Fig. 4, we fix the number of channels $K$ to 16 , and we illustrate the packetblocking probabilities with respect to the wavelength-conversion ratio $r$ for different values of the tuning-range ratio $d$ and for two values of the load $\rho$; the upper set of curves is for $\rho=0.5$ and the lower set for $\rho=0.25$ for all the figures presented in this paper. Independent of the conversion policy used, we observe improved performance with increasing tuning range. We also observe that for small tuning-range ratios, it is not as necessary to use large wavelength-conversion ratios as in the case of large tuning-range ratios. As expected, the three policies provide similar results for both small and large values of $d$, whereas for moderate values of $d$, the far-conversion policy outperforms the random-conversion policy, which again outperforms the nearconversion policy. This outperformance is apparent especially for large conversion ratios where the packet losses are mostly due to range occupancy and not due to the lack of converters. The analytical model, on the other hand, provides a good approximation only when the tuning-range ratio is large, but it provides a reference lower bound for all the cases studied. In the second example, we fix $K=32$ and investigate the performance of the three conversion policies for four different pairs of $W$ and $\rho$ values in Fig. 5, which clearly demonstrates that the approximate analysis method provides a lower bound for all three policies and can also be very effective in approximating the far-conversion behavior. We also observe that the impact of the far-conversion policy is stronger with increased wavelength-conversion ratios, moderate tuningrange ratios, and for lighter loads.

We now compare the circular and noncircular schemes employing far-, near-, and random-conversion policies in Figs. 6(a)-6(d) for two different values $d$ and $\rho$. We 


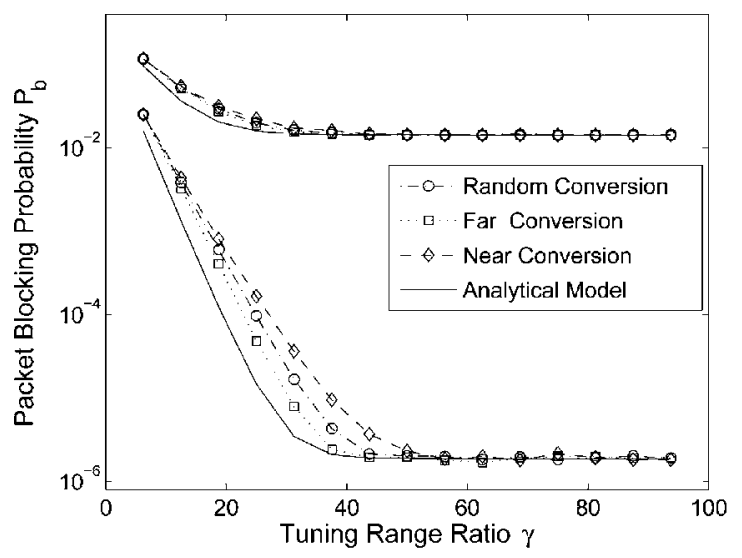

(a) $W=15$

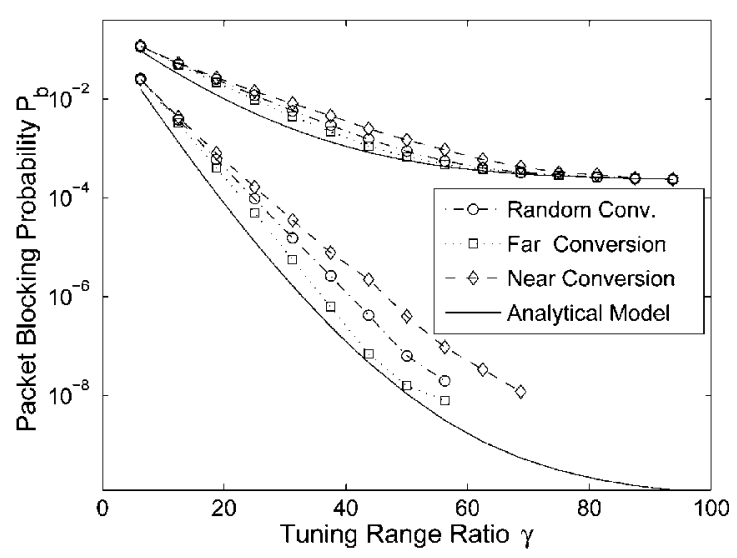

(b) $W=25$

Fig. 5. Blocking probabilities for $K=32$ with respect to $\gamma$ for two different values of $\rho=0.25$ and $\rho$ $=0.5$ when (a) $W=15$ and (b) $W=25$.

observe that the circular scheme always gives a better performance than the noncircular scheme, which is obvious since the conversion ranges are reduced for the latter scheme. We also observe that the far-conversion policy outperforms the others for both conversion schemes. It is also clear from these figures that the blocking probabilities stay intact when a certain conversion ratio is reached. This means that for larger conversion ratios, all losses are due to the fullness of the tuning range and adding new converters is not beneficial in this regime.

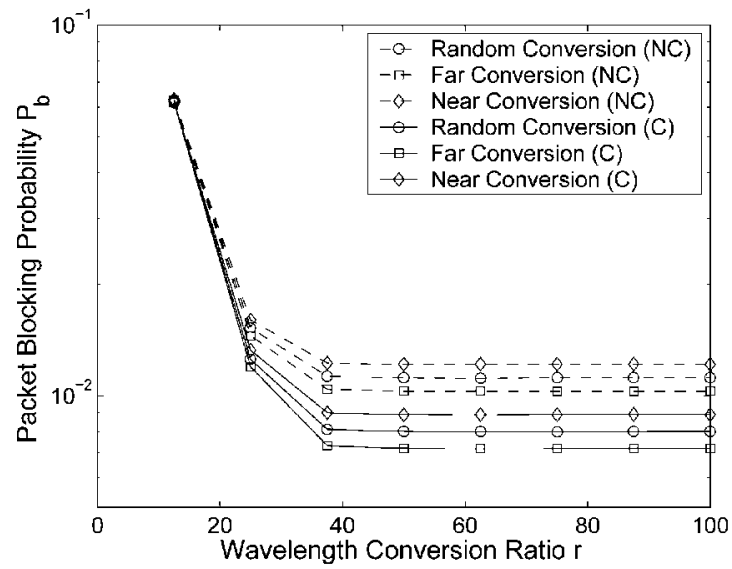

(a) $d=4, \rho=0.3$

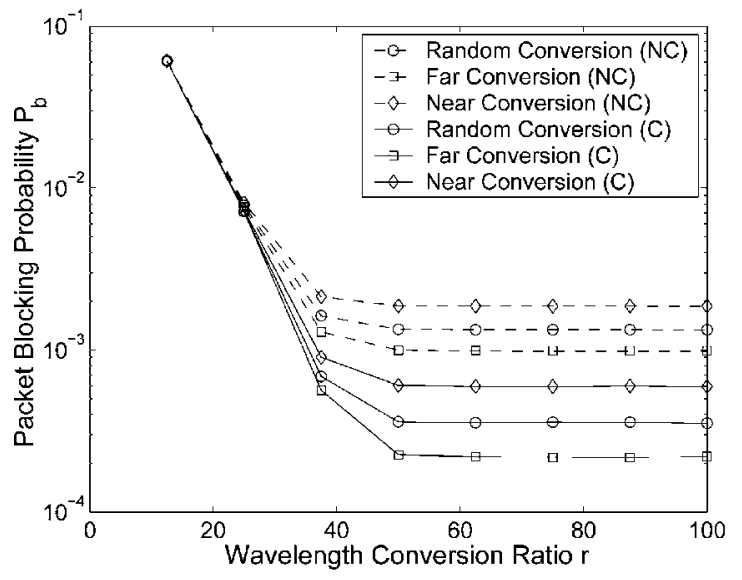

(c) $d=8, \rho=0.3$

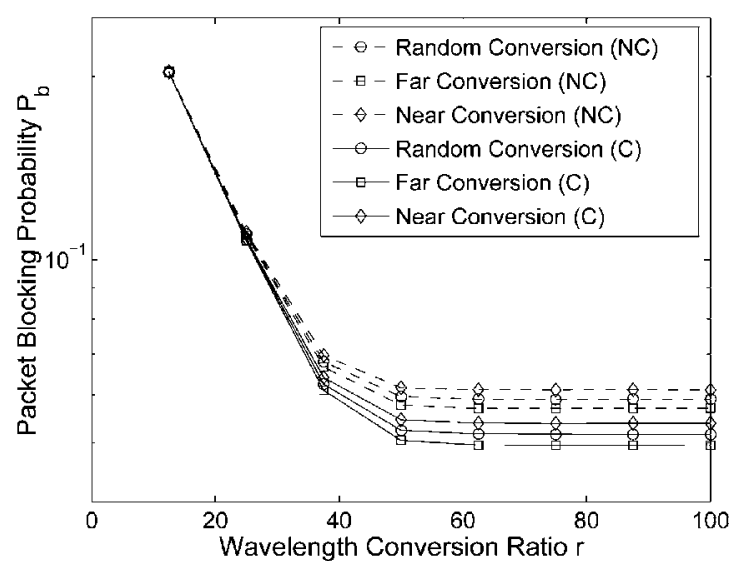

(b) $d=4, \rho=0.5$

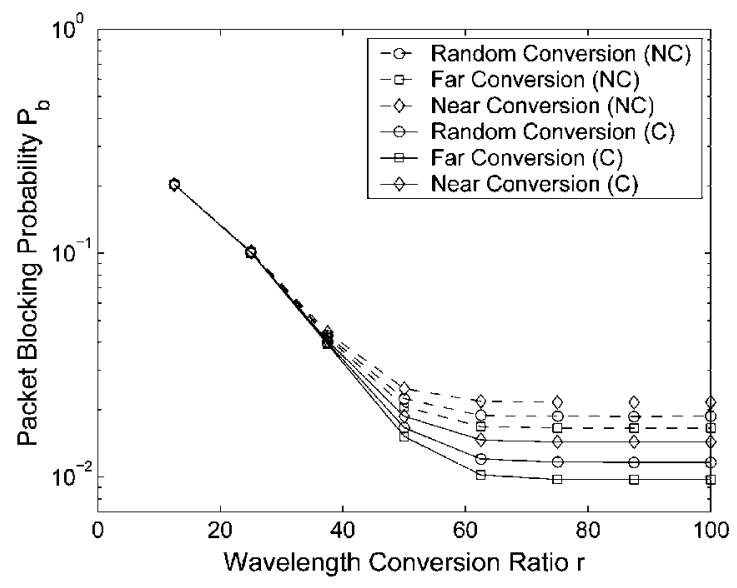

(d) $d=8, \rho=0.5$

Fig. 6. Blocking probabilities in circular (C)- and noncircular (NC)-conversion regimes for $K=32$ with respect to $r$ and (a) $d=4, \rho=0.3$, (b) $d=4, \rho=0.5$, (c) $d=8, \rho=0.3$, and $d=8, \rho=0.5$. 


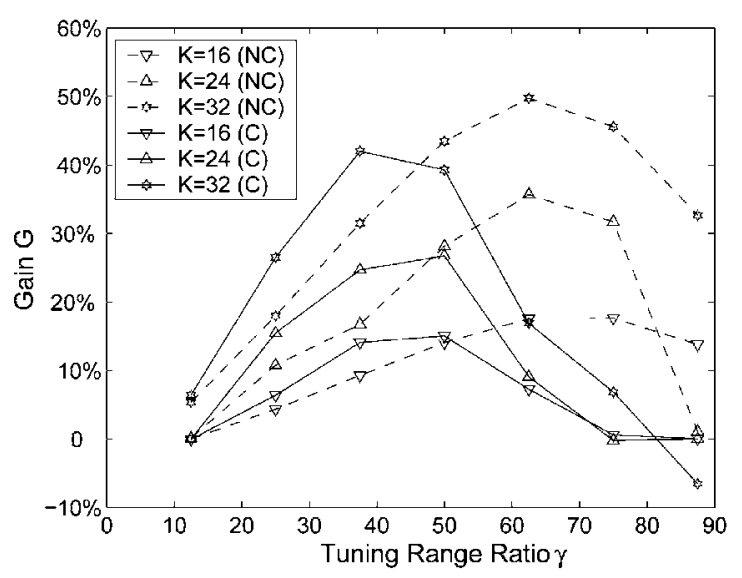

(a) $W=K$

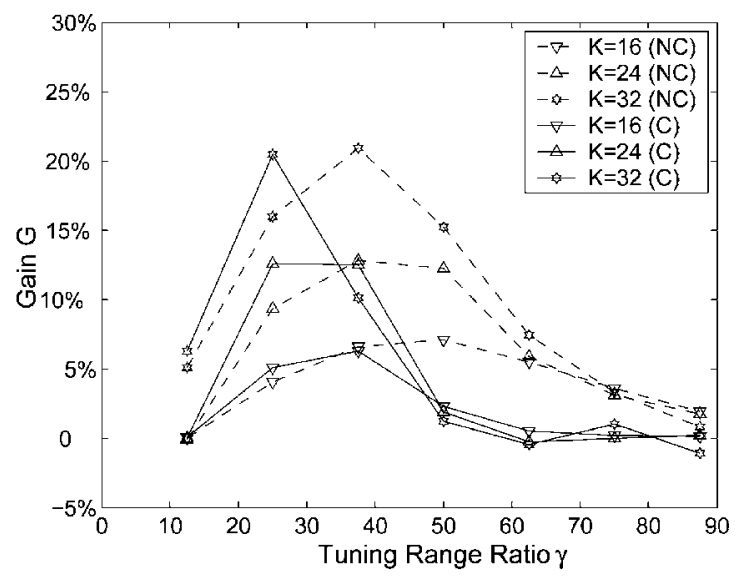

(b) $W=K / 2$

Fig. 7. Gain $G$ plotted as a function of $\gamma$ for circular- and noncircular conversion schemes for $\rho=0.4$ and different values of $K$, (a) FWC case $(W=K)$ and (b) $W=K / 2$. Curves referred to as (C) and (NC) correspond to circular and noncircular schemes, respectively.

To quantify the advantage in using far conversion, we introduce a normalized percentage gain parameter called $G$, which is defined through

$$
G=100 \frac{P_{b}(\text { random conversion })-P_{b}(\text { far conversion })}{P_{b}(\text { random conversion })} .
$$

We study the gain $G$ in blocking probabilities in using far conversion relative to random conversion with respect to $\gamma$ for circular- and noncircular-conversion schemes in Fig. 7. For this example, we fix $\rho=0.4$ and use three different values of $K, K$ $=16,24,32$. The FWC case and $W=K / 2$ cases are depicted in Figs. $7(\mathrm{a})$ and $7(\mathrm{~b})$, respectively. Let us first focus on the FWC case. For a fixed $K$, the gain first increases with increasing tuning-range ratio and peaks at a certain ratio and then drops to zero for both circular and noncircular schemes. This result is as expected, since it is clear that wavelength-conversion policies do not have any impact on the two boundary regimes, namely, $d=2$ and $d=K-1$. Another crucial observation is that the maximum achievable gain increases with increased $K$, so we expect to see the impact of far conversion more substantially in larger systems. As an example, an approximately $42 \%$ gain is achievable for $K=32$ and $d=12$. The regime in which the maximum gain is achievable turns out to include moderate tuning-range ratios, i.e., $35<\gamma<55$. When noncircular conversion is applied, we obtain similar behavior except that the gains appear to be shifted to the right, leading us to believe that the maximum gains are achievable this time for larger tuning-range ratios, $55<\gamma<75$. Otherwise, we still observe up to $50 \%$ gains even with noncircular conversion. Let us now take a look at the $W=K / 2$ case in Fig. 7(b). It is clear that the gains are reduced in this case when compared with the FWC case. The explanation for this phenomenon is that with fewer converters, most losses are due to lack of converters, and wavelength conversion policies have reduced impact on packet-blocking performance.

\section{Conclusions}

In this paper, we study the performance of asynchronous optical packet switching nodes equipped with a number of limited-range (LR) tunable wavelength converters shared on a per-output-link basis. We identify the wavelength clustering effect to describe the spatial correlation among the occupied wavelengths, which is detrimental to blocking performance in systems with LR converters. We propose a far-conversion policy to reduce the clustering effect, and we show through simulations that this proposed policy notably outperforms the other random- and near-conversion policies especially in the low load and moderate tuning-range ratio regimes. We also introduce an auxiliary Markovian model that captures the effect of the tuning range but not the clustering phenomenon. We show through simulations that this simplified model provides a lower bound for the packet-blocking probabilities for the three studied wavelength-conversion policies. 


\section{Acknowledgments}

This work is supported in part by The Science and Research Council of Turkey (Tübitak) under grant EEEAG-106E046 and by the Commission of the European Community IST-FP6 e-Photon/ONe project.

\section{References}

1. P. Gambini, M. Renaud, C. Guillemot, F. Callegati, I. Andonovic, B. Bostica, D. Chiaroni, G. Corazza, S. L. Danielsen, P. Gravey, P. B. Hansen, M. Henry, C. Janz, A. Kloch, R. Krahenbuhl, C. Raffaelli, M. Schilling, A. Talneau, and L. Zucchelli, "Transparent optical packet switching: network architecture and demonstrators in the KEOPS project," IEEE J. Sel. Areas Commun. 16, 1245-1259 (1998).

2. G. N. Rouskas and L. Xu, "Optical packet switching," in Emerging Optical Network Technologies: Architectures. Protocols, and Performance, K. Sivalingam and S. Subramaniam, eds. (Springer, 2004), pp. 111-127.

3. C. Qiao and M. Yoo, "Optical burst switching (OBS) - a new paradigm for an optical Internet,” J. High Speed Networks 8, 69-84 (1999).

4. Y. Chen, C. Qiao, and X. Yu, "Optical burst switching: a new area in optical networking research,” IEEE Network Mag. 18, 16-23 (2004).

5. V. Eramo, M. Listanti, and P. Pacifici, "A comparison study on the wavelength converters number needed in synchronous and asynchronous all-optical switching axchitectures," J. Lightwave Technol. 21, 340-355 (2003).

6. N. Akar and E. Karasan, "Exact calculation of blocking probabilities for bufferless optical burst switched links with partial wavelength conversion," in 1st Conference on Broadband Networks (BROADNETS'04), Optical Networking Symposium (IEEE, 2004), pp. 110-117.

7. V. Puttasubbappa and H. Perros, "An approximate queueing model for limited-range wavelength conversion in an OBS switch," in Networking (2005).

8. V. Eramo, M. Listanti, and M. Spaziani, "Resources sharing in optical packet switches with limited-range wavelength converters," J. Lightwave Technol. 23, 671-687 (2005).

9. N. Akar, E. Karasan, and K. Dogan, "Wavelength converter sharing in asynchronous optical packet/burst switching: an exact blocking analysis for Markovian arrivals," Part Supplement, IEEE J. Sel. Areas Commun. 24, 69-80 (2006).

10. J. Yates, J. Lacey, D. Everitt, and M. Summerfield, "Limited-range wavelength translation in all-optical networks," in INFOCOM (3) (IEEE, 1996), pp. 954-961.

11. T. Tripathi and K. N. Sivarajan, "Computing approximate blocking probabilities in wavelength routed all-optical networks with limited-range wavelength conversion," IEEE J. Sel. Areas Commun. 18, 2123-2129 (2000).

12. Z. Zhang and Y. Yang, "Performance modeling of bufferless WDM packet switching networks with limited-range wavelength conversion," IEEE Trans. Commun. 54, 1473-1480 (2006).

13. V. Puttasubbappa and H. Perros, "Performance analysis of limited-range wavelength conversion in an OBS switch," Telecommunications Systems Journal 31, 227-246 (2006).

14. L. Kleinrock, Queuing Systems, Vol. 1. Theory (Wiley, 1975).

15. G. H. Golub and C. F. van Loan, Matrix Computations, 3rd ed. (Johns Hopkins U. Press, 1996).

16. D. Gross and C. M. Harris, Fundamentals of Queueing Theory, 3rd ed. (Wiley, 1998). 